\title{
Polarisations of quasi-waves in a general anisotropic porous solid saturated with viscous fluid
}

\author{
M D SHARMA \\ Department of Mathematics, Kurukshetra University, India 136119. \\ e-mail: mohan_here@rediffmail.com
}

\begin{abstract}
Wave propagation is studied in a general anisotropic poroelastic solid saturated with a viscous fluid flowing through its pores of anisotropic permeability. The extended version of Biot's theory is used to derive a system of modified Christoffel equations for the propagation of plane harmonic waves in such media. The non-trivial solution of this system is ensured by a biquadratic equation whose roots represent the complex velocities of four attenuating quasi-waves in the medium. These complex velocities define phase velocity and attenuation of each quasi-wave propagating along a given phase direction in three-dimensional space. The solution itself defines the polarisations of the quasi-waves along with phase shift. The variations of polarisations of quasi-waves with their phase direction, are computed for a realistic numerical model.
\end{abstract}

\section{Introduction}

The mechanical behaviour of porous media is important in the seismic exploration, specially, for the closer description of physical phenomena around the oil reservoirs. To infer hydraulic transport properties of reservoir rocks from seismic data is a difficult but important aspect of exploration studies. A reservoir is, no doubt, a fluid-saturated porous solid medium pervaded by aligned cracks. In the presence of aligned cracks, an elastic medium behaves anisotropic to wave propagation (Crampin 1981). Reservoir rocks also exhibit a strong anisotropy in permeability of pores (i.e., hydraulic anisotropy). The porosity and permeability are the fundamental parameters that are economically important in oil production. The porosity of a solid is the most important geometrical property to hold a fluid. The permeability is an equally important physical property of a porous medium to conduct fluid flow in its pores.

The full-dynamic theory for wave propagation in fluid-saturated porous media was developed by Biot (1956). Biot used Lagrange's equations to derive a set of coupled differential equations that govern the motions of solid and fluid phases. Biot (1962a) extended the acoustic propagation theory in the wider context of the mechanics of porous media. Biot (1962b) developed new features of the extended theory, in more detail. This theory is obtained through a new and simplified derivation of the fundamental equations of poroelastic propagation. This also provides an exact procedure for the evaluation of the dynamic properties of the fluid motion relative to the solid. Since then, most of the studies on propagation in porous media are based on Biot's theories. The presence of viscosity in the pore-fluid and its effects on wave phenomena are also studied. Deresiewicz and Rice (1962) studied reflection of plane waves in the elastic frame of porous solid saturated with a viscous fluid. Sharma and Gogna (1991) discussed wave propagation in porous solid with a viscoelastic frame filled with a viscous fluid. In recent years, Gurevich (2002) discussed the elastic wave attenuation in porous rock saturated with viscous porefluid. For the anisotropic propagation, the author (Sharma 2004a) used Biot's 1956 theory to study the phase velocities and attenuations of quasiwaves in a general anisotropic porous solid with

Keywords. Anisotropy; poroelasticity; polarisation; attenuation. 
anisotropic permeability controlling the flow of viscous fluid in its pores. In another paper (Sharma 2004b), author used Biot's 1956 theory to study velocities and polarisation in anisotropic porous solid saturated with non-viscous fluid. The present study, also, discusses the polarisations of quasiwaves in poroelastic solid but with viscous fluid saturating the porous solid. The presence of viscosity in saturating fluid results in frequency dependent propagation. Moreover, mathematical model for wave propagation in dissipative porous medium is derived from the Biot theory of 1962 which, as said earlier, is a refined and extended version of the Biot 1956 theory. Effects of symmetries in hydraulic and elastic anisotropies on polarisations of quasi-waves are studied numerically, for different frequency regimes of Biot's theory.

Slowness (or velocity) and polarisation are two important tools to study the wave propagation properties in anisotropic media. The velocity anisotropy is often considered to be an obvious indicator of anisotropy. However, the velocity variations which are averaged over very long paths are not of much use in studying a local structure. Moreover, the complicated structure of the earth inhibits our ability to obtain accurate measurements of velocity over a range of directions in a material. This makes velocity anisotropy difficult to observe. An easily observable and distinctive feature of wave propagation in anisotropic solids is the deviation of particle displacements from the ray path. The polarisations of direct arrivals are more sensitive to the local properties of a medium. So, within a few wave-lengths of the recording station, the diagnostic effect of anisotropy is better represented by the polarisation anomalies than spatial dispersion of velocities. These anomalies contain information about the nature of anisotropic alignments and in some cases may indicate the depth of the anisotropic layer. Some of the studies relating the polarisation vectors to the physical and dynamical properties of the elastic media are discussed in Sharma (2004b).

\section{Anisotropic poroelastic propagation: Biot (1962) theory}

Following Biot (1962a, b), a set of differential equations governs the particle motion in a general anisotropic porous solid frame saturated by a viscous fluid in its pores of anisotropic permeability. These equations, in the absence of body forces, are given by

$$
\begin{aligned}
c_{i j k l}^{\prime} u_{k, j l}+m_{i j} w_{k, j k} & =\rho \ddot{u}_{i}+\rho_{f} \ddot{w}_{i}, \\
m_{k j} u_{k, i j}+R w_{k, i k} & =\rho_{f} \ddot{u}_{i}+q_{i j} \ddot{w}_{j}+\mu r_{i j} \dot{w}_{j},
\end{aligned}
$$

where $u_{i}$ are the components of the average displacements for the solid and $w_{i}$ are the components of displacement of fluid relative to the solid. The repeated (dummy) index implies summation, dot represents (partial) time derivative and comma before an index denotes partial space differentiation. The elastic constants of the porous aggregate, $c_{i j k l}^{\prime}$, are related to the elastic constants of porous matrix $\left(c_{i j k l}\right)$, by

$$
c_{i j k l}^{\prime}=c_{i j k l}+f\left(m_{i j} \delta_{k l}+m_{k l} \delta_{i j}-f R \delta_{i j} \delta_{k l}\right) .
$$

The elastic constant $R$ measures the pressure to be exerted on the fluid to push its unit volume into the porous matrix. The elastic $\left(m_{i j}\right)$ and inertial $\left(q_{i j}\right)$ parameters control the anisotropic coupling between fluid and solid phases. Another tensor $r_{i j}$ steers the generalized Darcy's law for fluid-solid coupling and represents the inverse of anisotropic permeability $\left(\chi_{i j}\right)$ tensor. All these tensors are symmetric. The $\rho$ and $\rho_{f}$ are densities of porous aggregate and pore fluid, respectively. Depending upon frequency $(\omega)$, viscosity $(\mu)$ and permeability tensor $\chi$, dissipation is represented by a matrix

$$
\mathbf{d}=\imath \frac{\mu}{\omega \rho_{f} \chi_{o}}\left\{\chi_{\mathbf{a}}\right\}^{-1}
$$

where, $\chi_{o}$ is normalization factor of permeability tensor $\chi=\chi_{o} \chi_{\mathbf{a}} \cdot \chi_{\mathbf{a}}$ is the normalized anisotropic permeability tensor (Rasolofosaon and Zinszner 2002). This expression of $\mathbf{d}$ is valid, only, for the low-frequency range, where the flow in the pores are of Poiseuille type. For higher frequencies, a correction factor (Deresiewicz and Rice 1962) is applied to dynamic viscosity $\mu$, replacing it by $\mu F(\kappa)$. With $\bar{a}$ denoting the linear dimension of pores, the $\kappa=\bar{a} \sqrt{\omega \rho_{f} / \mu} . F(\kappa)$, a complex function of frequency $\omega$, is defined through different expressions for $\kappa \ll 1$ (i.e., highly viscous fluid and/or smaller pores) and $\kappa \gg 1$ (i.e., low viscosity fluid and/or wider pores).

For the propagation of plane waves, consider the harmonic solution

$$
\begin{aligned}
& u_{j}=S_{j} \exp \left\{i \omega\left(p_{k} x_{k}-t\right)\right\}, \quad(j=1,2,3), \\
& w_{j}=F_{j} \exp \left\{i \omega\left(p_{k} x_{k}-t\right)\right\}, \quad(j=1,2,3),
\end{aligned}
$$

where, $\omega$ is angular frequency and $\left(p_{1}, p_{2}, p_{3}\right)$ is slowness vector. In terms of phase velocity $v$, the slowness $\left(p_{1}, p_{2}, p_{3}\right)=\mathbf{N} / v$, where, the real row matrix $\mathbf{N}=\left(n_{1}, n_{2}, n_{3}\right)$ represents the direction of phase propagation for homogeneous waves. Substituting (4) in (1), yields a system of six homogeneous equations in $S_{1}, S_{2}, S_{3}, F_{1}, F_{2}, F_{3}$. This larger system is manipulated (Sharma 2004a), 

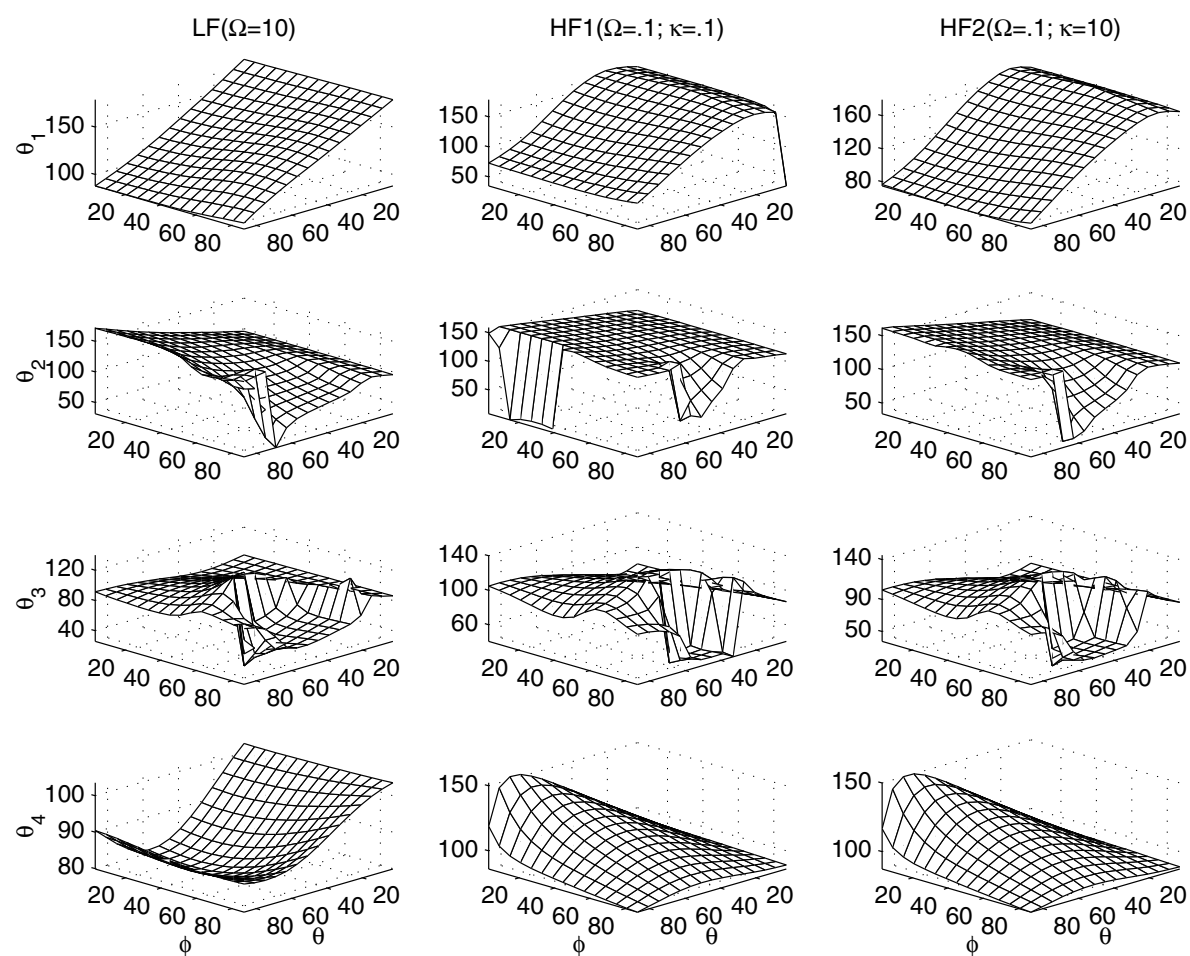

Figure 1(a). Polar angle $\left(\theta_{j}\right)$ variations of fluid discharge in dissipative dolomite (anisotropic poroelastic medium) with phase direction $(\theta, \phi)$, in different frequency regimes (LF: $\Omega=10 ; \mathrm{HF} 1: \Omega=0.1, \kappa=0.1 ; \mathrm{HF} 2: \Omega=0.1, \kappa=10$ ); all angles are in degrees.

to get a system of Christoffel equations, given by $\left(\right.$ for $\left.h=(1 / R) \rho_{f} v^{2}\right)$

$$
\begin{gathered}
W_{i j} S_{j}=0, \quad(i=1,2,3) \\
\mathbf{W}=\mathbf{D}_{1} h+\mathbf{D}_{2}+\frac{\mathbf{D}_{3}}{d_{0} h+d_{1}}+\frac{\mathbf{D}_{4}}{h\left(d_{0} h+d_{1}\right)}
\end{gathered}
$$

and an expression (for $\left.\mathbf{M}=(1 / R)\left\{m_{i j}\right\}\right)$,

$$
\begin{aligned}
w_{i}= & \Gamma_{i j} u_{j}, \quad(i=1,2,3) ; \\
\boldsymbol{\Gamma}= & \frac{1}{d_{o} h+d_{1}}\left[-\mathbf{\Phi} h+\mathbf{\Phi} \mathbf{N}^{\prime} \mathbf{N} \mathbf{M}\right. \\
& \left.-\mathbf{\Psi}+\frac{1}{h} \mathbf{\Psi} \mathbf{N}^{\prime} \mathbf{N M}\right],
\end{aligned}
$$

relating the relative fluid displacement $(\mathbf{w})$ to the solid displacement $(\mathbf{u})$. The coefficient matrices $\mathbf{D}_{\mathbf{j}},(j=1,2,3,4)$, are explained as follows:

$$
\begin{aligned}
& \mathbf{D}_{\mathbf{1}}=-\frac{\rho}{\rho_{f}} \mathbf{I}+\frac{1}{d_{o}} \mathbf{X}_{\mathbf{1}} \\
& \mathbf{D}_{\mathbf{2}}=\mathbf{Z}+\frac{1}{d_{o}}\left(\mathbf{X}_{\mathbf{2}}-\frac{d_{1}}{d_{o}} \mathbf{X}_{\mathbf{1}}\right)
\end{aligned}
$$

$$
\mathbf{D}_{\mathbf{3}}=\mathbf{X}_{\mathbf{3}}-\frac{d_{1}}{d_{o}}\left(\mathbf{X}_{2}-\frac{d_{1}}{d_{o}} \mathbf{X}_{\mathbf{1}}\right)
$$

$$
\mathbf{D}_{4}=\mathrm{MN}^{\prime} \mathbf{N} \Psi \mathbf{N}^{\prime} \mathrm{NM}
$$

The symmetric matrix $\mathbf{Z}=\left\{c_{i j k l}^{\prime} n_{j} n_{l}\right\}$. The dissipation matrix $\mathbf{d}$ is used to define another symmetric matrix

$$
\mathbf{Y}=\frac{1}{\rho_{f}} \mathbf{q}+\mathbf{d},
$$

where, matrix $\mathbf{q}=\left\{q_{i j}\right\}$. The elements of symmetric matrix $\boldsymbol{\Psi}$, are, then, obtained in cyclic order from two of its elements given by

$$
\Psi_{11}=2 Y_{23} n_{2} n_{3}-Y_{22} n_{3}^{2}-Y_{33} n_{2}^{2}
$$

and

$$
\Psi_{23}=Y_{23} n_{1}^{2}+Y_{11} n_{2} n_{3}-Y_{21} n_{3} n_{1}-Y_{31} n_{2} n_{1} .
$$

Other variables are expressed as follows:

$$
\begin{gathered}
d_{o}=\operatorname{det}(\mathbf{Y}) ; \quad \boldsymbol{\Phi}=\operatorname{adj}(\mathbf{Y}) ; \quad d_{1}=-\mathbf{N} \boldsymbol{\Phi} \mathbf{N}^{\prime} \\
\mathbf{X}_{\mathbf{1}}=\mathbf{\Phi} ; \quad \mathbf{X}_{\mathbf{2}}=\mathbf{\Psi}-\left(\mathbf{M} \mathbf{N}^{\prime} \mathbf{N} \boldsymbol{\Phi}+\boldsymbol{\Phi} \mathbf{N}^{\prime} \mathbf{N M}\right) \\
\mathbf{X}_{\mathbf{3}}=-\left(\mathbf{M} \mathbf{N}^{\prime} \mathbf{N} \boldsymbol{\Psi}+\mathbf{\Psi} \mathbf{N}^{\prime} \mathbf{N} \mathbf{M}\right)+\mathbf{M N}^{\prime} \mathbf{N} \boldsymbol{\Phi} \mathbf{N}^{\prime} \mathbf{N M}
\end{gathered}
$$


$\operatorname{LF}(\Omega=10)$
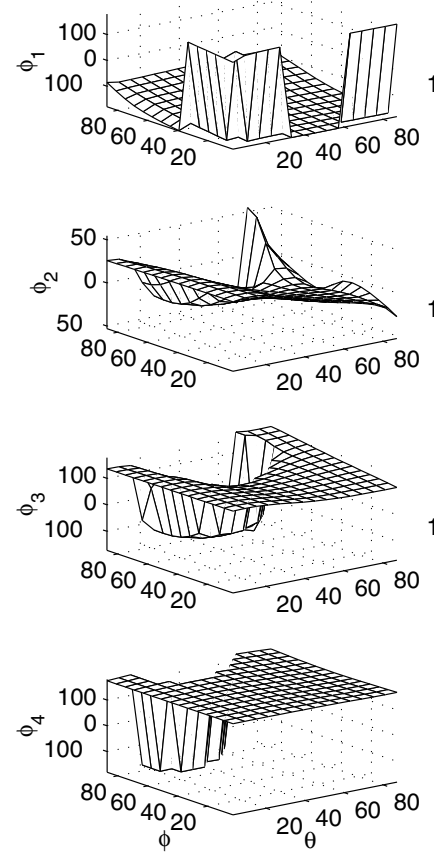

$\operatorname{HF} 1(\Omega=.1 ; \kappa=.1)$
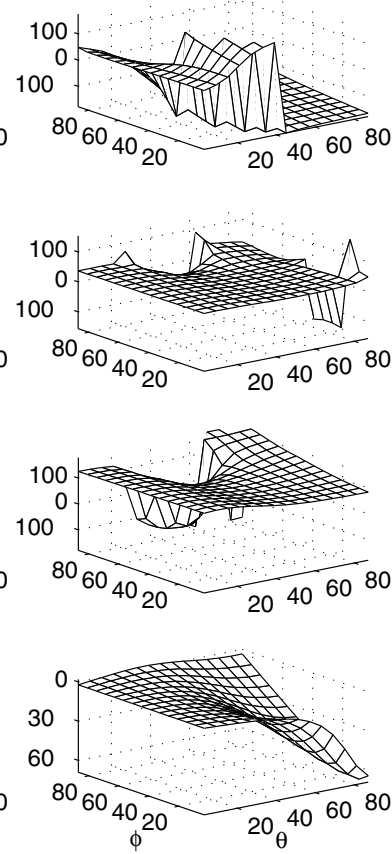

$\operatorname{HF} 2(\Omega=.1 ; \kappa=10)$
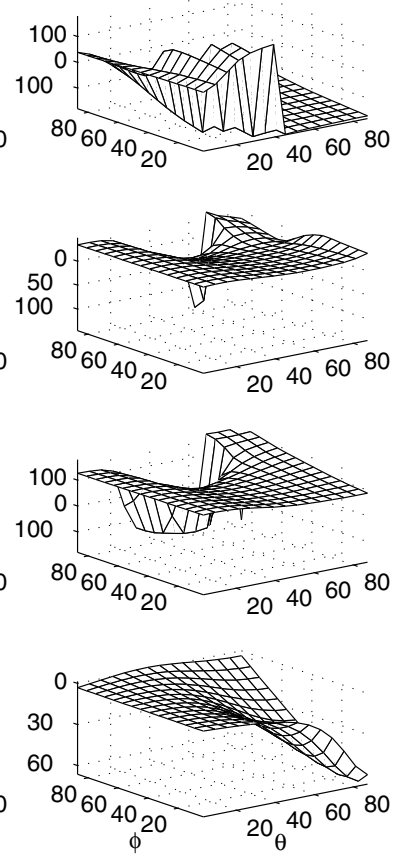

Figure 1(b). Azimuth $\left(\phi_{j}\right)$ variations of fluid discharge in dissipative dolomite with phase direction $(\theta, \phi)$, in different frequency regimes (LF: $\Omega=10$; HF1: $\Omega=0.1, \kappa=0.1$; HF2: $\Omega=0.10, \kappa=10$ ); all angles are in degrees.

In terms of characteristic frequency $\left(\omega_{c}\right)$ of Biot's theory, the dissipation matrix is written as

$$
\mathbf{d}=\imath \frac{\Omega}{f} \chi_{a}^{-1},
$$

where, the dimensionless parameter $\Omega=\left(\omega_{c} / \omega\right)$ decides the frequency regime. The value of $\Omega \gg$ 1 ensures the Poiseuille flow of pore-fluid and represents the low frequency regimes of Biot's theory. The high-frequency regime is represented by $\Omega \ll 1$. The non-trivial solution of the system of equations (5) is ensured by a complex biquadratic equation in $h=\left(\rho_{f} v^{2} / R\right)$. The four complex roots of this equation define the existence of four attenuating quasi-waves in the dissipative poroelastic solid. Let $h_{j},(j=1,2,3,4)$, denote the roots of this equation. The complex phase velocities of the four waves, given by $v_{j}=$ $\sqrt{\left(R h_{j} / \rho_{22}\right)},(j=1,2,3,4)$, will be varying with the direction of phase propagation. The complex velocity of a quasi-wave ' $j$ ', i.e., $v_{j}=$ $v_{R}+\imath v_{I}$, defines the phase propagation velocity $V_{j}=\left(v_{R}^{2}+v_{I}^{2}\right) / v_{R}$ and attenuation quality factor $Q_{j}^{-1}=-2 v_{I} / v_{R}$ for the corresponding wave.

\section{Polarisation}

For a quasi-wave of complex velocity $v$, the system of homogeneous complex equations (5) is solved for a non-trivial solution. Such a solution, in the form $\left(S_{1}, S_{2}, S_{3}\right)$, defines the polarisation of this quasiwave. The complex components of polarisation vector are resolved as $S_{j}=R_{j} e^{\imath \xi_{j}},(j=1,2,3)$. The non-zero real values of $\xi_{j}$ implies that the plane of constant phase is shifted by $\left(\xi_{1}, \xi_{2}, \xi_{3}\right)$ in three-dimensional space. The displacement components in the solid phase are, now, written as:

$u_{j}=R_{j} \exp \left[\imath \omega\left(\frac{1}{v} n_{k} x_{k}-t\right)+\imath \xi_{j}\right] ; \quad(j=1,2,3)$.

Taking the real parts of the expressions on the right side of these equations, the polarisation of the quasi-wave is represented by a vector $\left(P_{1}, P_{2}, P_{3}\right)$. Its components are given by

$$
\begin{aligned}
& P_{j}=R_{j} \cos \left(\xi_{j}+X\right), \quad(j=1,2,3) ; \\
& X=\omega\left(\frac{v_{R}}{v_{R}^{2}+v_{I}^{2}} n_{k} x_{k}-t\right)
\end{aligned}
$$

where, $v_{R}$ and $v_{I}$ are real and imaginary parts of complex velocity $v$ of the quasi-wave. The polarisations of quasi-waves vary with the direction of phase propagation $\mathbf{N}$. The averaged particle motion (w) of the fluid particles flowing out of porous matrix is expressed through a polarisation vector $\left(Q_{1}, Q_{2}, Q_{3}\right)$, given by 

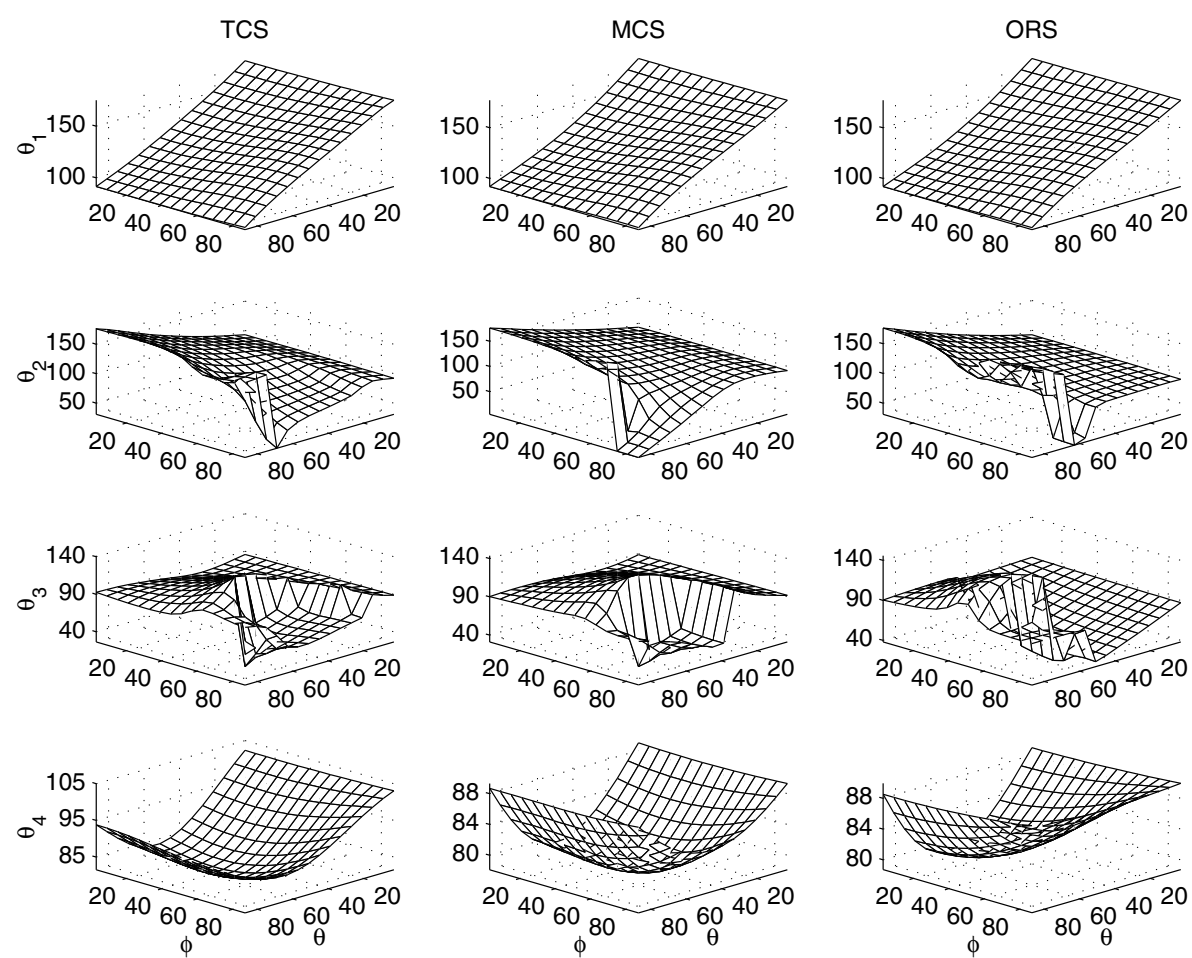

Figure 2(a). Effect of elastic anisotropy on the polar angle variations $\left(\theta_{j}\right)$ of pore-fluid discharge with phase direction $(\theta, \phi)$, in dissipative dolomite (TCS: $Z_{1}=Z_{2}=1$; MCS: $Z_{1}=0, Z_{2}=1$; ORS: $Z_{1}=Z_{2}=0$; Regime: LF); all angles are in degrees.

$$
\begin{aligned}
Q_{j}= & \sum_{k=1}^{3} R_{k}\left[R e\left(\Gamma_{j k}\right) \cos \left(\xi_{k}+X\right)\right. \\
& \left.+\operatorname{Im}\left(\Gamma_{j k}\right) \sin \left(\xi_{k}+X\right)\right] .
\end{aligned}
$$

The wave propagation in anisotropic poroelastic medium is not similar to that in anisotropic elastic medium. For instance, the propagation vectors of quasi-waves in anisotropic porous medium are not the eigen-vectors of a real symmetric positive definite matrix. This implies that these polarisation vectors may not, necessarily, be orthogonal to each other. Moreover, the presence of viscosity in the pore-fluid is accompanied by attenuation and dissipation. In a porous medium, the motion of fluid particles is different from solid particles and it varies with the phase direction when the solid is anisotropic. These variations can only be analyzed numerically for a particular model of anisotropic porous solid.

\section{Numerical computation and discussion}

The analytical expressions derived in section 2 define a general mathematical model for homogeneous wave propagation in anisotropic porous solid saturated with a viscous fluid. Section 3 explains the polarisations of quasi-waves in a dissipative medium. The expressions in these sections can be combined to compute the effects of

- elastic anisotropy (different symmetries) of poroelastic solid;

- viscosity of pore fluid;

- hydraulic (permeability) anisotropy of different symmetries;

- size and shape of pores;

- porosity of saturated porous solid;

- wave frequency;

on the polarisations of quasi-waves in the poroelastic medium. The present numerical work is, however, restricted to study the effects of viscosity (through frequency regimes) and symmetries in elastic and hydraulic anisotropies. Triclinic, monoclinic and orthorhombic symmetries are considered. To deflate the numerical output, the polarisation anomalies are computed only for the relative motion (i.e., w) between fluid and solid particles of the porous aggregate. The importance of this relative motion (i.e., drainage) lies with its direct relation to the hydraulic transport properties of porous rocks. The direction of polarisation vector $\left(Q_{1}, Q_{2}, Q_{3}\right)$, for the quasiwave ' $k$ ', is denoted by $\left(\theta_{k}, \phi_{k}\right)$, where $\theta_{k}$ represents the polar angle and $\phi_{k}$ is the azimuth 

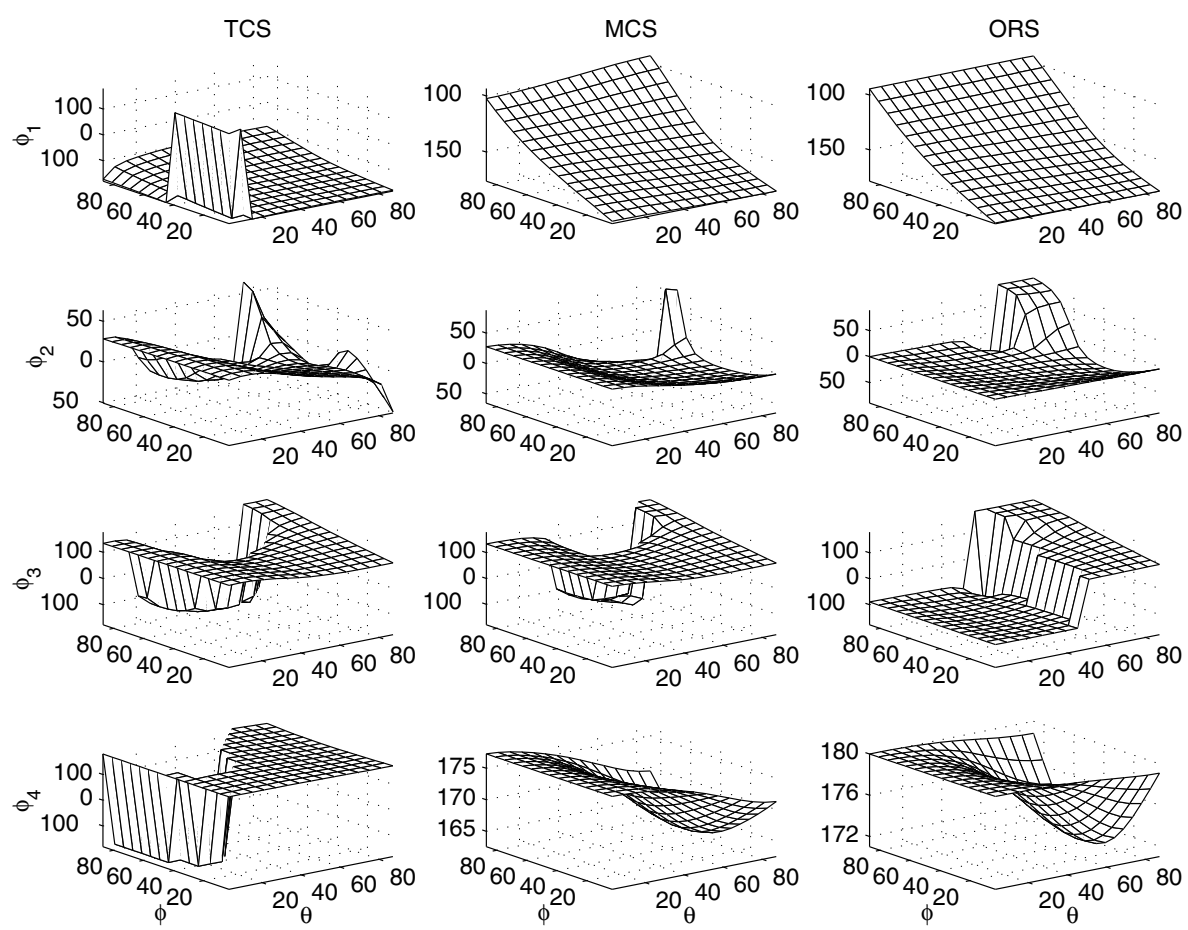

Figure 2(b). Effect of elastic anisotropy on azimuth variations $\left(\phi_{j}\right)$ of pore-fluid discharge with phase direction $(\theta, \phi)$, in dissipative dolomite (TCS: $Z_{1}=Z_{2}=1$; MCS: $Z_{1}=0, Z_{2}=1$; ORS: $Z_{1}=Z_{2}=0$; Regime: LF); all angles are in degrees.

with 1-direction. The polarisation anomalies in a real crystal may be a useful study. Elastic matrix (GPa) for Dolomite, an anisotropic reservoir rock, following Rasolofosaon and Zinszner (2002), is written as

$$
\begin{array}{lll}
c_{11}=65.53 & c_{12}=9.77 & c_{13}=12.19 ; \\
c_{14}=0.18 Z_{1} & c_{15}=-0.81 Z_{1} & c_{16}=2.94 Z_{2} ; \\
c_{22}=50.77 & c_{23}=11.61 & c_{24}=-0.09 Z_{1} ; \\
c_{25}=-0.50 Z_{1} & c_{26}=-0.19 Z_{2} & c_{33}=60.11 ; \\
c_{34}=-1.61 Z_{1} & c_{35}=1.78 Z_{1} & c_{36}=0.84 Z_{2} ; \\
c_{44}=23.51 & c_{45}=1.49 Z_{2} & c_{46}=-1.17 Z_{1} ; \\
c_{55}=24.57 & c_{56}=0.26 Z_{1} & c_{66}=20.21 .
\end{array}
$$

The density of this $23 \%$ porous material saturated with a fluid of density $980 \mathrm{~kg} / \mathrm{m}^{3}$ is $2423 \mathrm{~kg} / \mathrm{m}^{3}$. The values $(\mathrm{GPa})$ chosen for elastic coupling parameters are $\mathbf{M}=0.33 \mathbf{M}_{\mathbf{a}}$ and dynamic coupling is assumed to be given by $\mathbf{q}=\frac{\rho_{f}}{f} \mathbf{M}_{\mathbf{a}}$, where, the matrix $\mathbf{M}_{\mathbf{a}}=\left\{1,-0.1 Z_{2}, 0.2 Z_{1} ;-0.1 Z_{2}, 1.1\right.$, $\left.0.15 Z_{1} ; 0.2 Z_{1}-0.15 Z_{1}, 0.9\right\}$ represent a general anisotropic elastic coupling between fluid and solid constituents of porous aggregate. The value assumed for $R=10 \mathrm{GPa}$. The anisotropic permeability tensor for Dolomite is given by, $\chi_{a}=$ $\left\{0.96,-0.08 Z_{2},-0.06 Z_{1} ;-0.08 Z_{2}, 0.72,0.01 Z_{1}\right.$; $\left.-0.06 Z_{1}, \quad 0.01 Z_{1}, \quad 0.73\right\}$. Identity matrix for $\chi_{a}$ represents the isotropic permeability. These anisotropic tensors with value of $Z_{1}=Z_{2}=1$ define the triclinic system of anisotropy. The monoclinic symmetry is represented by $Z_{1}=0, Z_{2}=1$ and the values $Z_{1}=Z_{2}=0$ represent the orthorhombic symmetry.

In the low-frequency wave propagation regime of Biot's theory, the Poiseuille flow $\left(\omega \ll \omega_{c}\right)$ in pores is ensured by the value of $\Omega=10$. Similarly, $\Omega=0.1$ represents the high-frequency regime of Biot's theory. Three propagation regimes are identified. One is the low-frequency regime (LF). The high frequency regimes are divided into two parts, one (HF1) with $\kappa=0.1$ (i.e., smaller pores and/or high viscosity of pore-fluid) and other (HF2) with $\kappa=10$ (i.e., wider pores and/or low viscosity of pore-fluid).

Using the above numerical values, the directions, $\left(\theta_{k}, \phi_{k}\right)$, are computed for the four quasi-waves represented by $k=1,2,3,4$. The phase direction $(\theta, \phi)$ varies from $(0,0)$ to $\left(90^{\circ}, 90^{\circ}\right)$. Details are as follows.

\subsection{Polarisations in propagation regimes}

For the three propagation regimes, the polarisations $\left(\theta_{k}, \phi_{k}\right) ; k=1,2,3,4$, are computed for the 

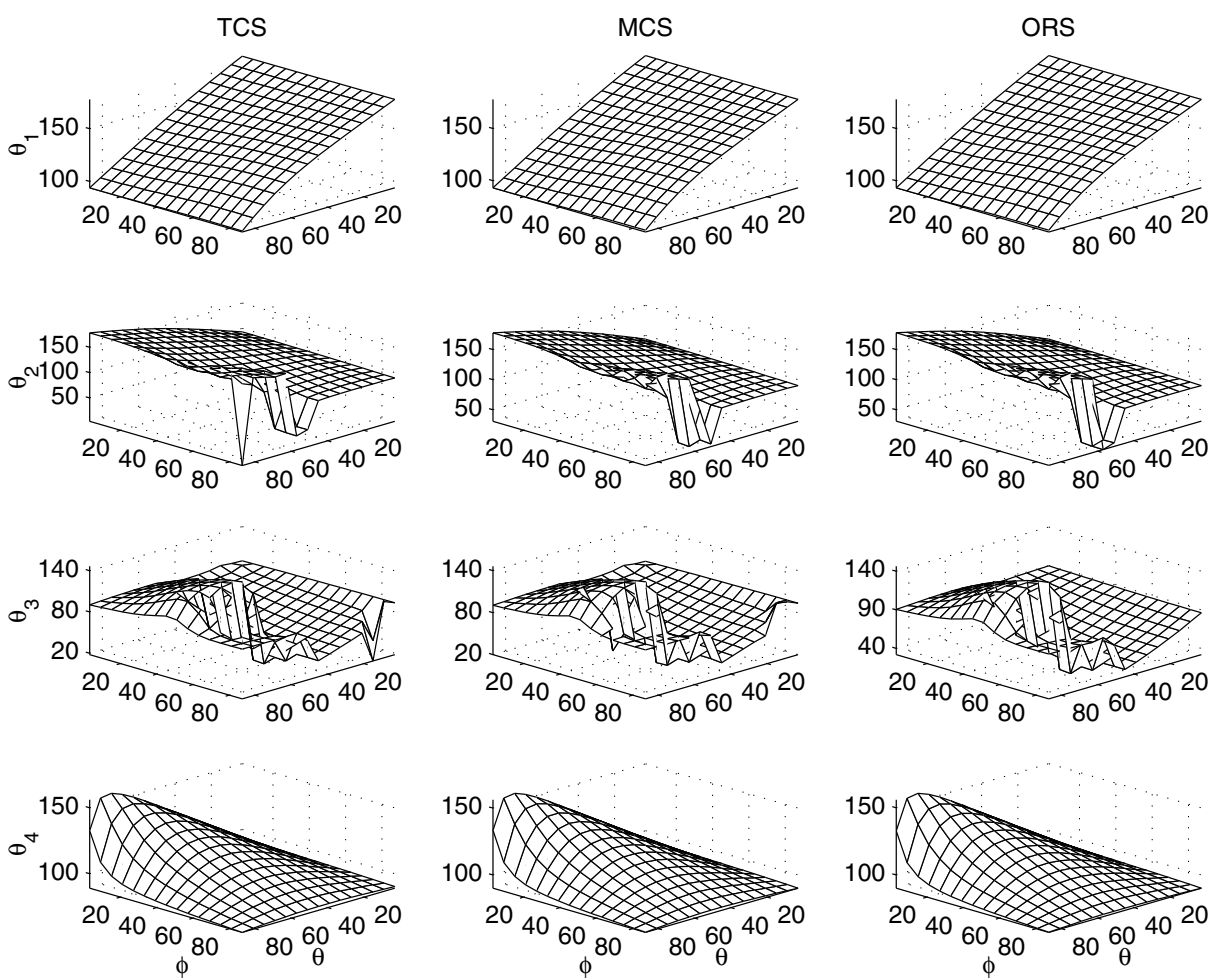

Figure 3(a). Effect of hydraulic anisotropy on the polar angle variations $\left(\theta_{j}\right)$ of pore-fluid discharge with phase direction $(\theta, \phi)$, in dissipative dolomite (TCS: $Z_{1}=Z_{2}=1$; MCS: $Z_{1}=0, Z_{2}=1$; ORS: $Z_{1}=Z_{2}=0$, Regime: HF1); all angles are in degrees.

four quasi-waves along the given phase directions $(\theta, \phi)$. Both, elastic and hydraulic, anisotropies are of general type (i.e., triclinic anisotropy). The variations in polar angles $\left(\theta_{k}\right)$ with phase direction are plotted in figure $1(\mathrm{a})$ and azimuth $\left(\phi_{k}\right)$ variations are plotted in figure 1(b). Going through the plots in these figures, it may be observed that frequency has little effect on the amount of polarisations but the effect is quite significant on the variations of the polarisation with phase direction. The slowest quasi-wave (i.e., $\theta_{4}, \phi_{4}$ ) is an exception in suffering most with the frequency change.

\subsection{Polarisations with elastic anisotropy}

The plots in figure 2(a) exhibit the polar angle variations with the phase direction in three major symmetries, (i.e., triclinic, monoclinic, orthorhombic), of elastic anisotropy. The corresponding variations in azimuths of quasi-waves are plotted in figure 2(b). The hydraulic anisotropy is of orthorhombic type. The propagation regime is LF. The effect of relaxing elastic anisotropy is observed more on the variations of polarisations and little on the magnitude. The slowest quasiwave, i.e., $\left(\theta_{4}, \phi_{4}\right)$, is the most sensitive to elastic anisotropy.

\subsection{Polarisations with hydraulic anisotropy}

The elastic anisotropy is with orthorhombic symmetry. The propagation regime is HF1. The polarisations are plotted for three major symmetries of hydraulic anisotropy. The plots in figure 3(a) exhibit polar angle variations and those, in figure 3(b), represent azimuth variations in the particle motions due to the propagation of each of the four quasi-waves. The plots in these figures may be observed to note only the small effect of hydraulic anisotropy on the magnitude and variations of polarisations.

\section{Conclusions}

The numerical results in the previous section were computed only for a particular but realistic model. The discussion of these results may not qualify for the quantitative generalization. To summarize the qualitative effects of anisotropy, frequency, and viscosity, the observations from the numerical results may be interpreted for the following conclusions.

- The particle motions of the quasi-waves are not even near to mutual orthogonal. 

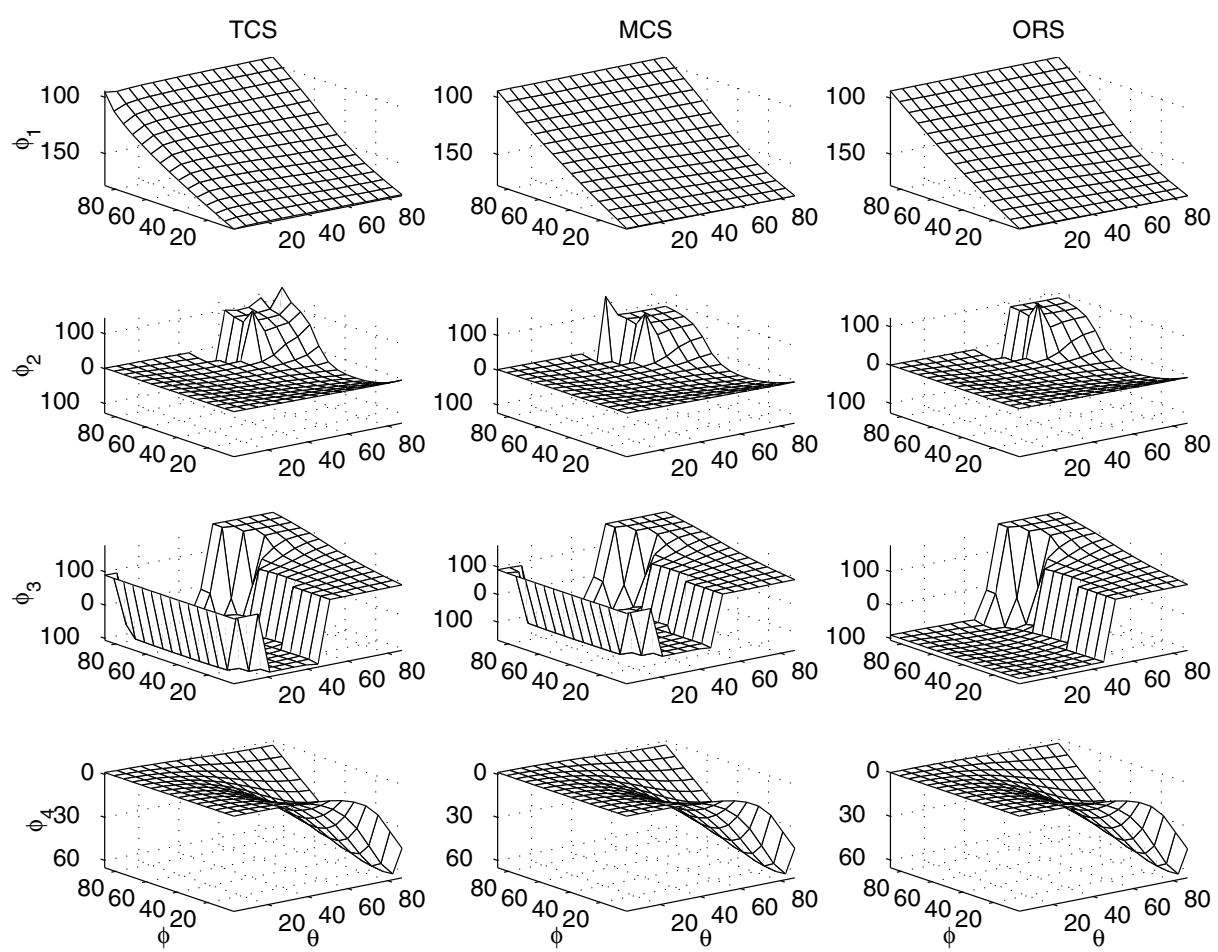

Figure 3(b). Effect of hydraulic anisotropy on azimuth variations $\left(\phi_{j}\right)$ of pore-fluid discharge with phase direction $(\theta, \phi)$, in dissipative dolomite (TCS: $Z_{1}=Z_{2}=1$; MCS: $Z_{1}=0, Z_{2}=1$; ORS: $Z_{1}=Z_{2}=0$ Regime: HF1); all angles are in degrees.

- The presence of anisotropy changes the relative motion between fluid and solid particles of porous aggregate. The particles of the two constituents will no longer be moving parallel to each other, for any of the quasi-wave.

- It is, perhaps, the quasi-wave corresponding to slow P-wave of Biot's theory, which is very sensitive to the anisotropy of porous frame and the wave-frequency.

- The directional difference between the motions of fluid and solid particles may be less sensitive to the symmetries of hydraulic anisotropy.

This work studies wave propagation in a realistic medium keeping in mind the general physical properties of porous rocks. The mathematical model derived relates the seismic properties of reservoir rocks to their lithological and production properties. This study may be helpful to prospective seismologists working to improve oil recovery. The difference in polarisations of two constituents of a poroelastic solid may help to diagnose the type and amount of anisotropy in porous rocks. The study of anisotropic poroelasticity may also be important for understanding the mechanical behaviour of composite materials leading to enormous applications in non-destructive testing/evaluation studies (Braga 1990; Buden and Datta 1990; Chai and $\mathrm{Wu}$ 1994; Wu and $\mathrm{Wu} 2000)$.

\section{References}

Biot M A 1956 The theory of propagation of elastic waves in a fluid-saturated porous solid, I. Low-frequency range, II. Higher frequency range; J. Acoust. Soc. Am. 28 168-191.

Biot M A 1962a Mechanics of deformation and acoustic propagation in porous media; J. Appl. Phys. 33 1482-1498.

Biot M A 1962b Generalized theory of acoustic propagation in porous dissipative media; J. Acoust. Soc. Am. $\mathbf{3 4}$ $1254-1264$.

Braga A M B 1990 Wave Propagation in Anisotropic Layered Composites, Ph.D. dissertation; Stanford University, Stanford, CA.

Buden M and Datta S K 1990 Rayleigh and love waves in cladded anisotropic media; ASME, J. Appl. Mech. $\mathbf{5 7}$ 398-403.

Chai J-F and Wu T-T 1994 Determinations of anisotropic elastic constants using laser-generated surface waves; J. Acoust. Soc. Am. 95 3232-3241.

Crampin S 1981 A review of wave motion in anisotropic and cracked elastic media; Wave Motion 3 343-391.

Deresiewicz H and Rice J T 1962 The effect of boundaries on wave propagation in a liquid-filled porous solid: III reflection of plane waves at a free plane boundary (general case); Bull. Seism. Soc. Am. 52 $595-625$

Gurevich B 2002 The effect of fluid viscosity on elastic wave attenuation in porous rocks; Geophysics 67 264-270.

Rasolofosaon P N J and Zinszner B E 2002 Comparison between permeability anisotropy and elasticity anisotropy of reservoir rocks; Geophysics 67 230-240.

Sharma M D 2004a Wave propagation in a general anisotropic poroelastic medium with anisotropic 
permeability: phase velocity and attenuation; Int. J. Solids Struct. 41 4587-4597.

Sharma M D 2004b Three-dimensional wave propagation in a general anisotropic poroelastic medium: phase velocity, group velocity and polarisation; Geophys. J. Int. 156 329-344.
Sharma M D and Gogna M L 1991 Seismic wave propagation in a viscoelastic porous solid saturated by viscous liquid; Pure and Appl. Geophysics 135 383-400.

$\mathrm{Wu}$ T-T and Wu T-Y 2000 Surface waves in coated anisotropic medium loaded with viscous fluid; $A S M E$, J. Appl. Mech. 67 262-266.

MS received 11 October 2004; accepted 7 February 2005 